\title{
CAN Based Vehicle Health Monitoring System
}

\author{
Swathi M C, Dilnavincent, Navya S.R, Muhajira U P, Usharani J* \\ Department of Computer Science and Engineering, GSSS Institute of Engineering and \\ Technology for Women, Mysuru, India
}

DOI: https://doi.org/10.21467/proceedings.1.23

* Corresponding author email: usharani@gsss.edu.in

\begin{abstract}
This paper deals with developing an embedded system for detecting the vehicle condition by monitoring the internal parameters that are used in evaluating the vehicle's current health condition. Traveler information plays a critical role in supporting safety, security, mobility, and in improving the reliability of travel. This traveler information can be a continuous data on performance of the vehicle and the status of its internal components. In this project, CAN is used for communicating between the ARDUINO and the connected sensors. CAN helps in monitoring sensor data. This device is basically based on ARDUINO system which is the heart of this project that controls vibration detection sensor; alcohol detection sensor, ultrasonic sensor and temperature sensor are used. These sensed data are transferred to the controller in the form of packet frames and by using GSM/GPS and public mobile network services all the information can be transferred to web server and mobile phone. Many systems are developed for sensormonitoring.
\end{abstract}

Index Terms- CAN, ARDUINO, MQ3, ECU

\section{INTRODUCTION}

This system is based on the widely used CAN bus technology, to extract the vehicle's status or fault information. The increasing complexity of automotive electronic control system makes automotive fault diagnosis and maintenance work more difficult. Transfer of large amount of data and exchange of different signals between electronic control systems on the bus vehicle is essential. Therefore, vehicle interior network came into existence. CAN (Controller Area Network, CAN); relying on its stability performance, low price and high reliability and realtime, has now been widely used in automotive internal network. GSM and GPS technology are used for sending information of vehicle status when it faults are detected. The GPS system will provide location and time. GPS provides accurate location and time information for an unlimited number of users in all weather, day and night, anywhere in the world. It has the advantages of a wide coverage, high accessing speed, charging according to the flow rate.

In recent years the safety and health is very important irrespective of human being or Machines, in our project we were try to explain and prove how car safety and health matters. So we were using CAN Communication protocol for ECU to ECU communication and

(C) 2018 Copyright held by the author(s). Published by AIJR Publisher in Proceedings of the $3^{\text {rd }}$ National Conference on Image Processing, Computing, Communication, Networking and Data Analytics (NCICCNDA 2018), April 28, 2018.

This is an open access article under Creative Commons Attribution-NonCommercial 4.0 International (CC BY-NC 4.0) license, which permits any non-commercial use, distribution, adaptation, and reproduction in any medium, as long as the original work is properly cited. ISBN: $978-81-936820-0-5$ 
there is devices called Sensor ECU and Dash board ECU, sensor ECU read the Temperature, Air Pressure, Quality of the Air inside the Vehicle, once the data fetching is done ECU push data to Dash board ECU for Process and Display, If vehicle met in to unexpected accidents the vehicle it 'self-send the sensor data and location of the Vehicle to responsible persons (Emergency contacts).

The main purpose of an accident avoidance system is to reduce the chances of collision and continuous monitoring of the health parameters of driver and also the vehicle environment. Speeding, drunk driving, sudden fall of pulse rate and seat belt adjustments all lead to accidents.

\section{RELATED WORK}

CAN: The Controller Area Network (the CAN bus) is a serial communications bus for realtime control applications operates at data rates of up to 1 Megabits per second and has excellent error detection and confinement capabilities. CAN was originally developed by the German company, Robert Bosch, for use in cars, to provide a cost-effective communications busforin-carelectronicsandasalternativetoexpensive, cumbersome and unreliable wiring looms and connectors. The car industry continues to use CAN for an increasing number of applications, but because of its proven reliability and robustness, CAN is now also being used in many other control applications. CAN or Controller Area Network is a robust industrial strength hardware and software protocol used to communicate between microcontrollers. It is very popular in modern automotive applications and is gaining popularity in industrial and home automationapplications.

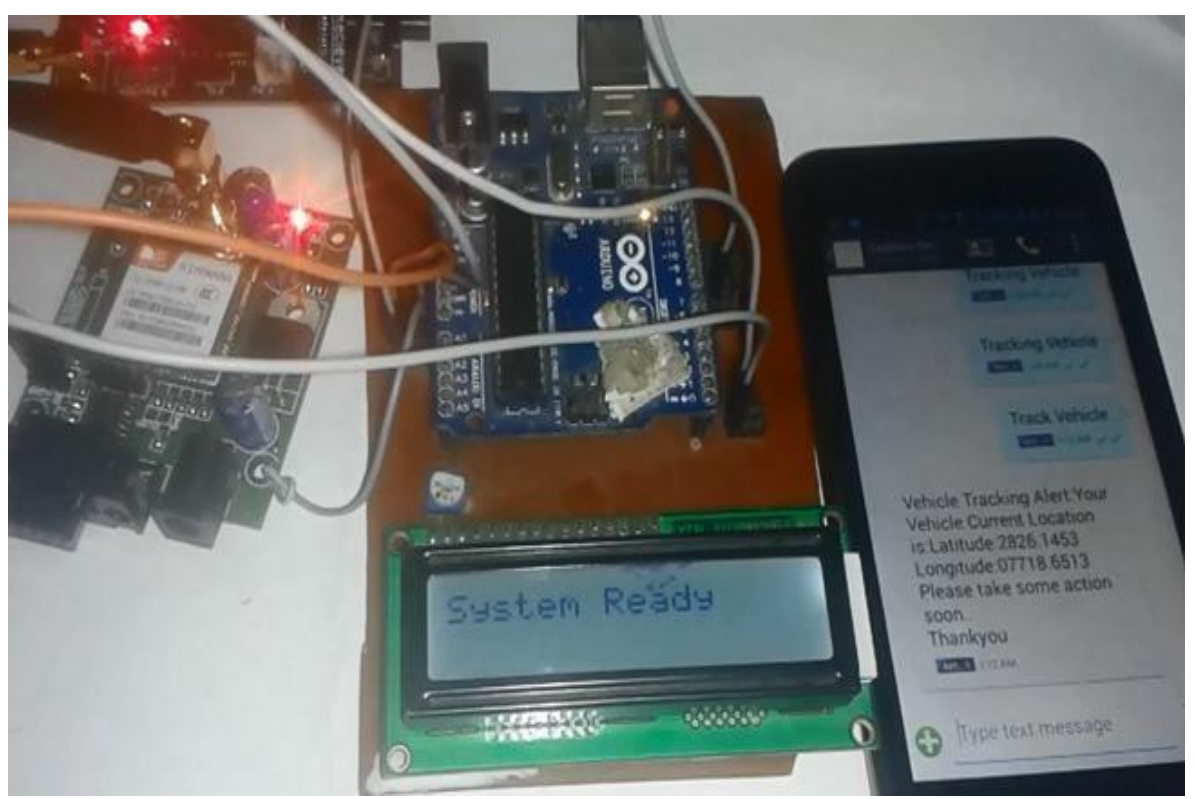

Figure 1: accident details tracking through mobile

Proceedings of the $3^{\text {rd }}$ National Conference on Image Processing, Computing, Communication, Networking and Data Analytics (NCICCNDA 2018) 
Working Principle: Data messages transmitted from any node on a CAN bus do not contain addresses of either the transmitting node, or of any intended receiving node.

\section{SYSTEMREQURIMENT}

To obtain the desired results we require a system which is reliable, secure and also efficient. The system requires compact package of hardware and software. It must full fill the necessary qualities such as real-time continuous monitoring and exact statistic series. It must support mobility and less powerconsumption

\subsection{Hardware architecture}

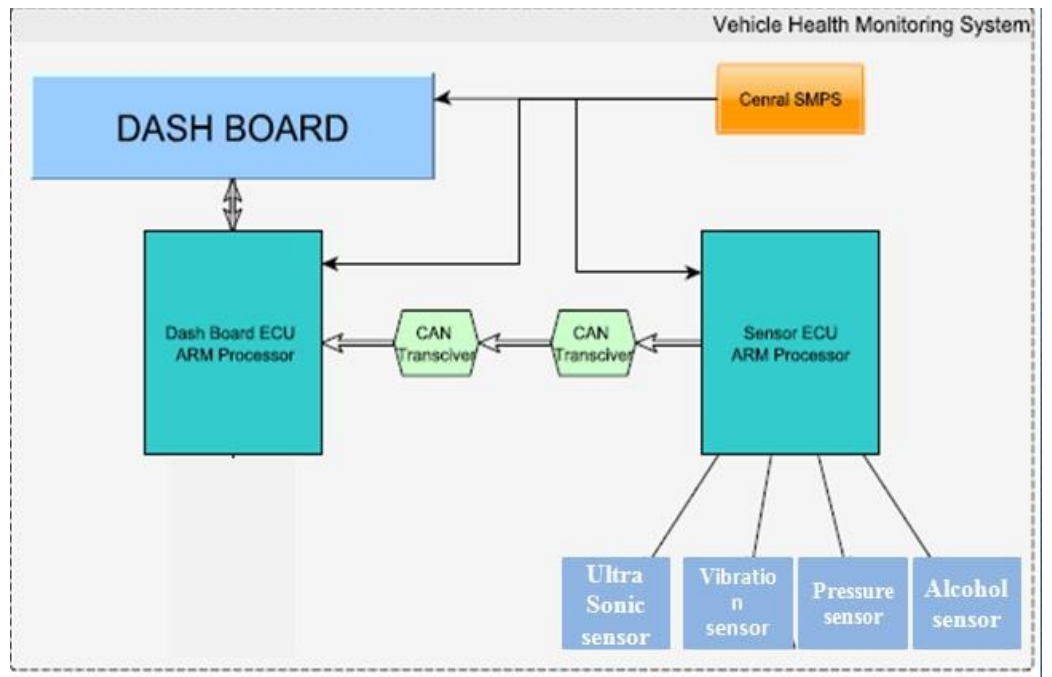

Figure 2: Block diagram of vehicle health monitoring system

The hardware structure mainly integrates the CAN bus controller, ARDUINO as the main control module, Temperature, Ultrasonic sensor (obstacle detecting sensor), Vibration sensor, alcohol sensor ,LCD displaytoprovide digital interface and other accessories. The block diagram of CAN based vehicle control system consist of ARDUINO controller and the engine control module which controls the vehicle status with various sensors. The controllers control the status of the vehicle and sends the feedback to operator panel by providing digitalinformation.

\subsection{Software Architecture}

The vehicle control system is programmed using the embedded C. software of the system have two parts one is program working with control module and another one is monitoring and logging of and running on the PC. After power supply and device driver initialization CAN control building function i.e. CAN init) etc. being initialized. Now read the sensor port processes the data and output it on the LCD screen and mobile device 


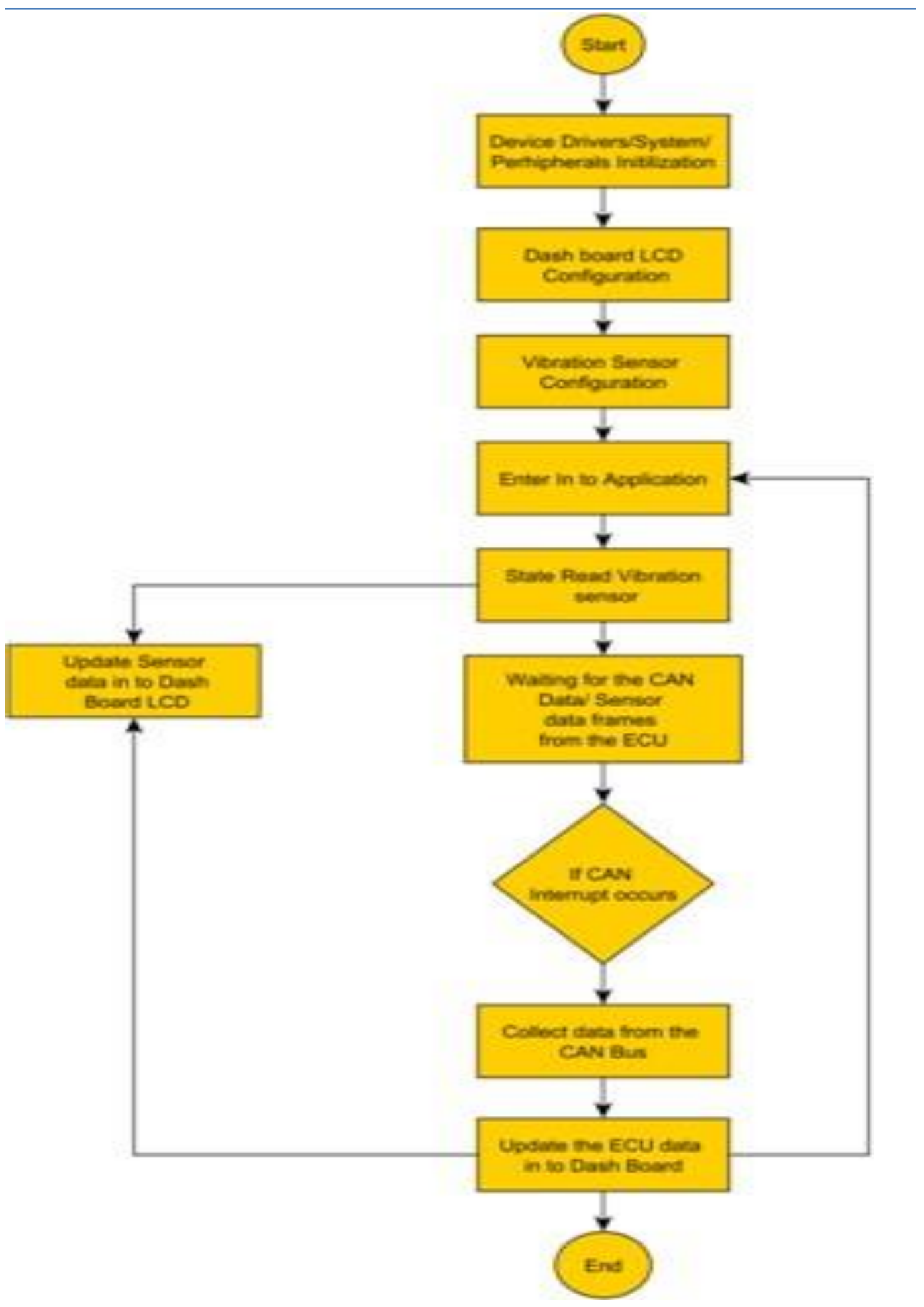

Figure 3: Flow chart

Proceedings of the $3^{\text {rd }}$ National Conference on Image Processing, Computing, Communication, Networking and Data Analytics (NCICCNDA 2018) 


\subsection{Sensors}

\subsubsection{Vibration detection sensor}

It is used to observe the collision of vehicle which is sensed due to vibration and shocks during accident. Sending the accident details to the nearby hospitals, police station and emergency contacts using GSM and GPSsupport.

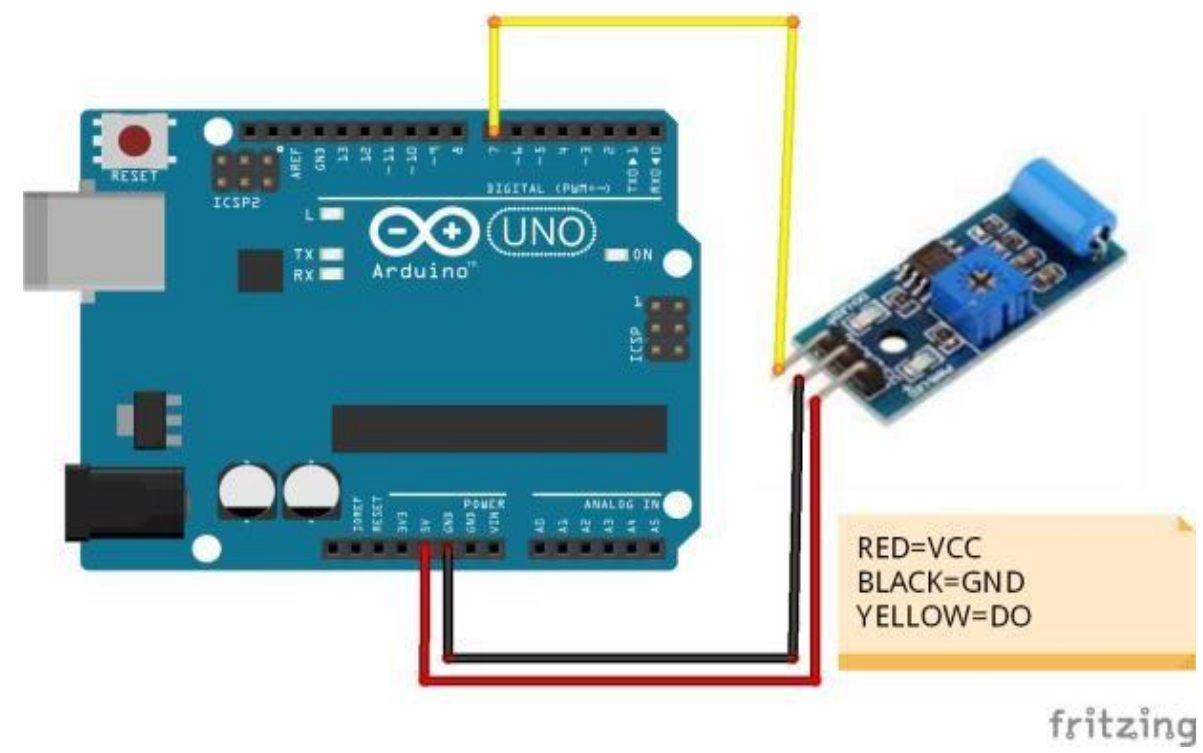

Figure 4: Vibration sensor

\subsubsection{Alcohol detection sensor}

Drowsiness of driver can cause mishap evasion that can be a reason of death. MQ3 sensor with breath analyzer is used here to check the liquor level.

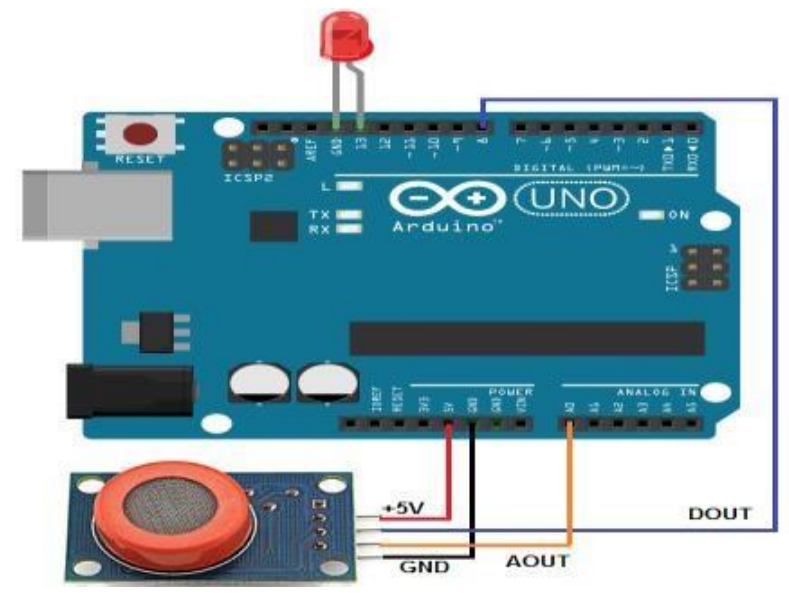

Figure 5: Alcohol sensor 


\subsubsection{Ultrasonic sensor}

It is used to detect the distances from obstacles thy col- lision can be avoided. It is based on the echolocation process. Transmitted sound waves bounced back and retrieved with some time difference that helps to calculate the distance.

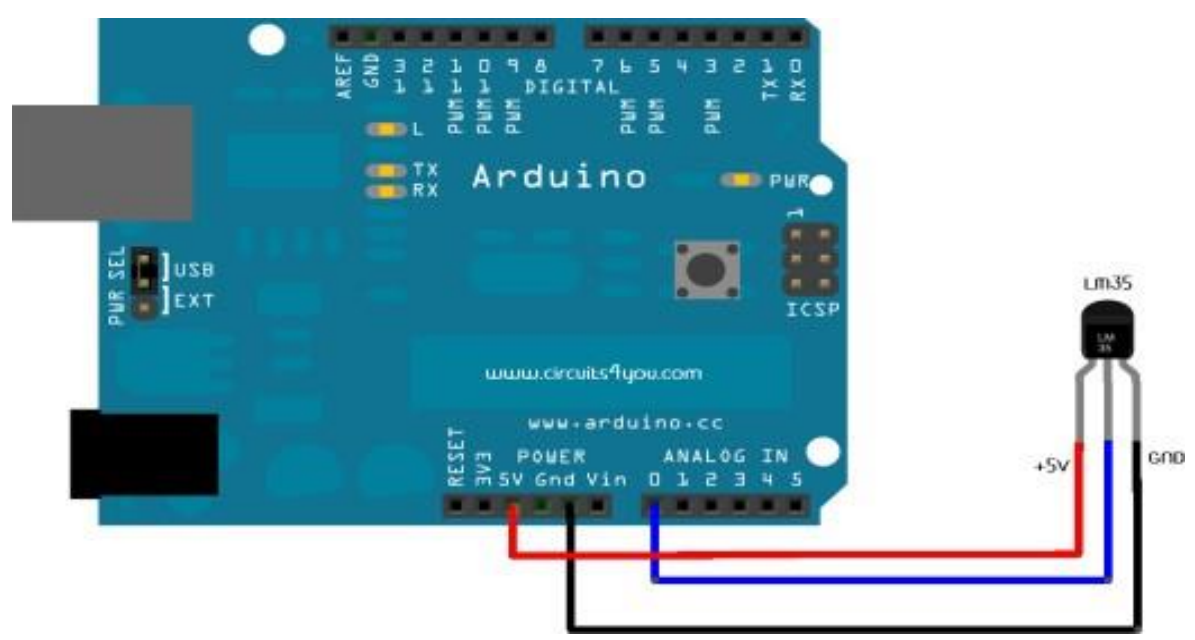

Figure 6: Ultrasonic sensor

\subsubsection{Temperature detection sensor}

The temperature sensor is used to measure the temperature of the engine of an internal combustion engine. The readings from this sensor are then fed back to the engine control unit (ECU), which uses this data to adjust the fuel injection and ignitiontiming.

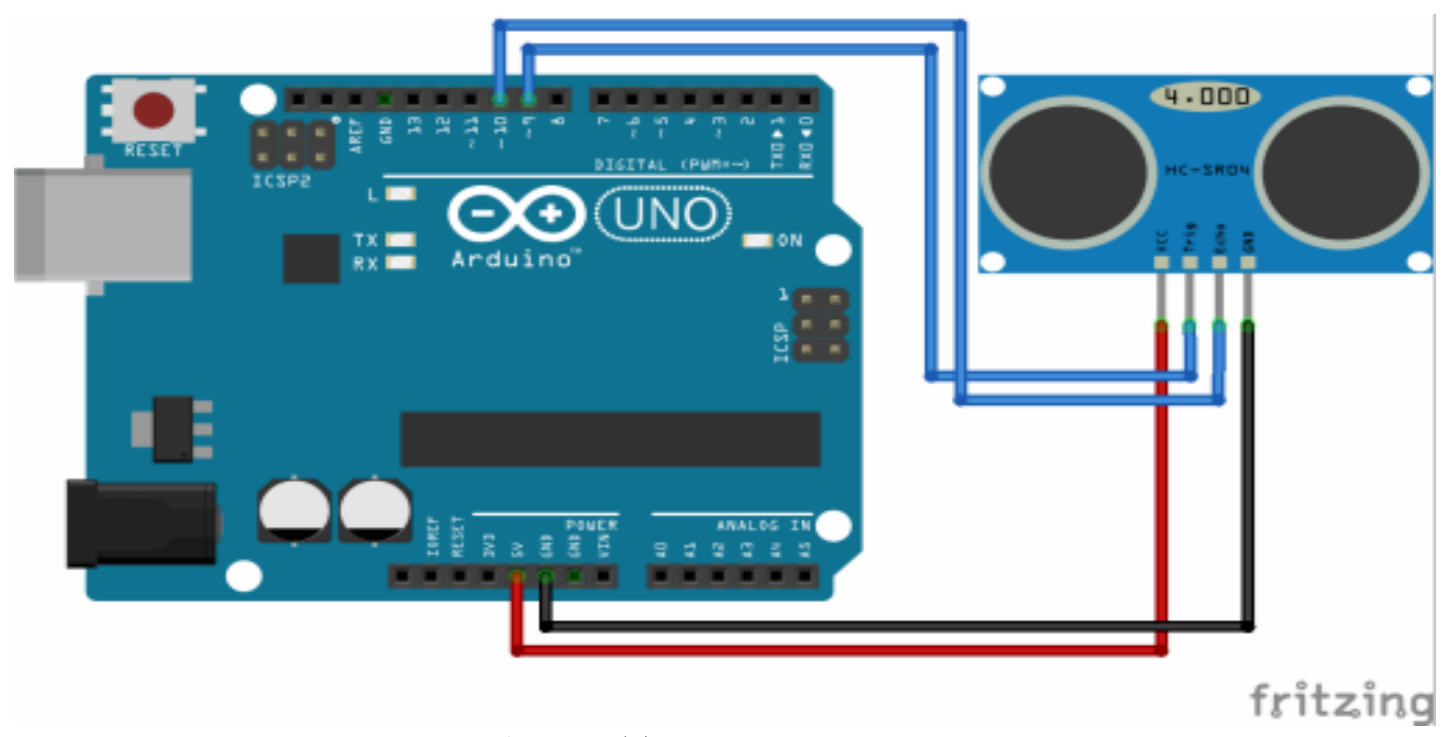

Figure 7: Temperature sensor

Proceedings of the $3^{\text {rd }}$ National Conference on Image Processing, Computing, Communication, Networking and Data Analytics (NCICCNDA 2018) 


\subsection{ARDUNIO}

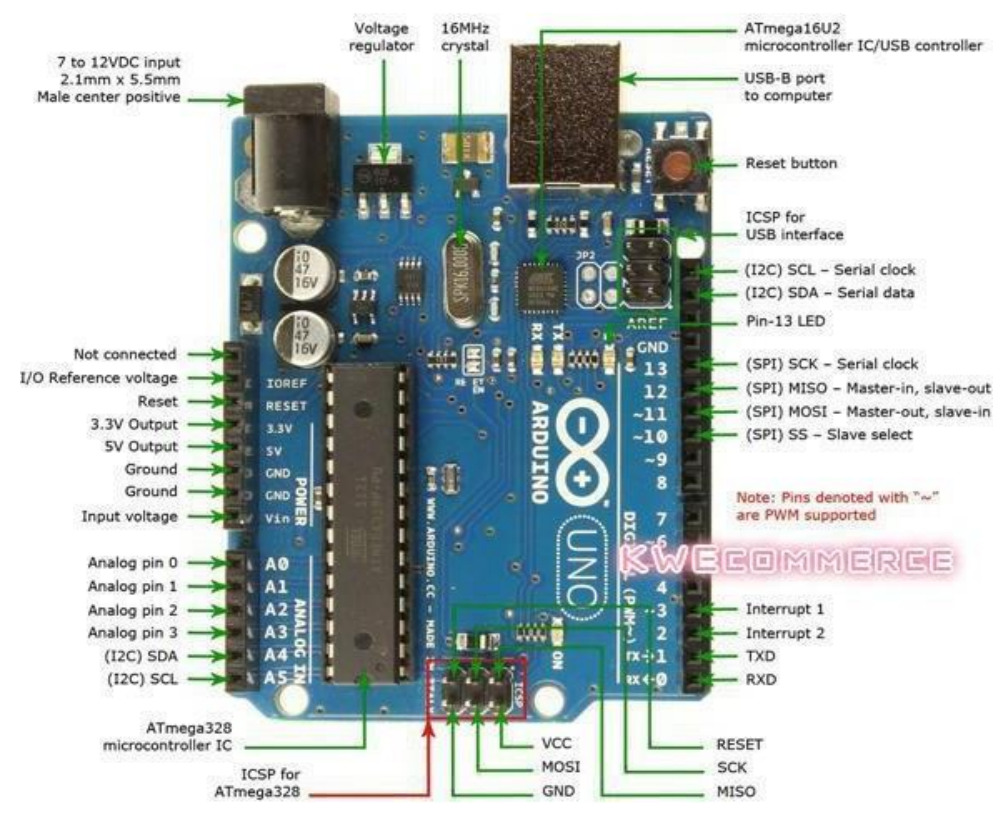

Figure 8: PIN configuration of ARDUINO UNO

The Arduino Uno is a microcontroller board based on the ATmega328 (datasheet). It has 14 digital input/output pins (of which 6 can be used as PWM outputs), 6 analog inputs, a $16 \mathrm{MHz}$ ceramic resonator, a USB connection, a power jack, an ICSP header, and a resetbutton.

\section{CONCLUSION}

The paper focuses on vehicle health monitoring system based on CAN bus, which can be used to improve the efficiency of monitoring, to maintain the system security, to lower the maintenance costs as well as the operating expensed. With the development of the mobile technology, the design will be made better; the data transmission based on wireless communication will be used more widely. Thecommunication network designed is able to stay stable in a long run in the experiment and meets the expected target. In future we can extend this project further by connecting more devices to make configuration of a vehicle health monitoring system more effective

\section{REFERENCE}

[1] P.Visconti,G.Cavalera "explain the intelligent system for monitoring and control of photovoltaic plants and for optimization of solar energy production"

[2] P.Costantini "proposes the design of electronic programmable board with user-friendly touch screen interface for management and control

[3] J Han,C.Choi WanKi Park llwoo Lee,"proposes the smart Home energy management system including renewable energy based on ZigBee and PLC"

[4] T.Obaid,H.Rashed,M.Tarique" explains the ZigBee Technology and its application I wireless Home Automation systems". 